\title{
A Hierarchically Tailored Wrinkled Three-Dimensional Foam for Enhanced Elastic Supercapacitor Electrodes
}

Siyeon Jang, ${ }^{\dagger}$ Hyeongho Min, ${ }^{\star}$ Sung Beom Cho,${ }^{\S}$ Hyeon Woo Kim, ${ }^{\S}$ Wonkyeong Son," Changsoon Choi," Sungwoo Chun ${ }^{* \wedge}$ and Changhyun Pang*t

${ }^{\dagger}$ School of Chemical Engineering, Sungkyunkwan University (SKKU), Seobu-ro, Jangan-gu, Suwon, 16419, Republic of Korea.

‡SKKU Advanced Institute of Nanotechnology, Sungkyunkwan University (SKKU), Seobu-ro, Jangan-gu, Suwon, 16419, Republic of Korea.

$\S$ Virtual Engneering Center, Korea Institute of Ceramic Engineering and Technology (KICET), Soho-ro 101, Jinju-si, Gyeonsangnam-do, 52851, Republic of Korea

"Department of Energy and Materials Engineering, Dongguk University-Seoul, Seoul 04620, Republic of Korea

${ }^{\wedge}$ Department of Electronics and Information Engineering, Korea University, Sejong 30019, Republic of Korea 
Corresponding authors: swchun127129@korea.ac.kr; chpang@skku.edu

\section{Experimental Section}

Fabrication of 3D PDMS foam. First, a polydimethylsiloxane (PDMS, Sylgard 184 Silicone Elastomer Kit, Dow Corning) (10wt\%) diluted solution was formed comprising liquid PDMS precursors mixing 10-parts base siloxane oligomers with 1-part curing agents and ethyl acetate (SAMCHUN CHEMICALS, South Korea) that serves as a solvent. The diluted solution was sufficiently blended using a vortex mixer for 30 min to achieve uniformity. Subsequently, nickel foam (SJMATERIALS, South Korea), with average pore size of $0.3 \mathrm{~mm}$ and thickness of $1.5 \mathrm{~mm}$, was coated with the PDMS solution within a vacuum chamber for 30 min by soaking in the solution to remove air bubbles trapped in the pores of the foam. The PDMS-coated Ni foam was removed from the solution and transferred into an oven set at $80{ }^{\circ} \mathrm{C}$, where it was thermally cured for $3 \mathrm{~h}$. Afterward, the foam coated with PDMS was immersed in a nickel etchant solution (UN2582 Ferric Chloride Solution, TRANSENE Company, Inc.) comprising iron (III) chloride $\left(\mathrm{FeCl}_{3}\right)$, which etches the nickel foam selectively for $48 \mathrm{~h}$. Cleaning stages to remove the etchant residue were accompanied by a process in which PDMS foam was dipped into diluted water for over an hour. Finally, 3D PDMS foam was obtained after drying in the oven at $80{ }^{\circ} \mathrm{C}$.

Formation of wrinkles on the surface of 3D PDMS foam. To modify the surface structure of the PDMS foam, a basic plasma cleaner equipment (PDC-32G Cleaner, Harrick Plasma, NY, USA) was used for the wrinkling process. The setup of the plasma equipment was fixed with a high frequency of $8-12 \mathrm{MHz}(\mathrm{RF})$, a power setting of $18 \mathrm{~W}$, and an inner pressure of $\approx 0.27$ mbar. PDMS foam was stretched by tensile strains of $10 \%, 20 \%, 30 \%$, and $40 \%$, and vitrified by oxygen plasma exposure simultaneously, allowing struts of the foam to constitute a bilayer that oxidized on the surface. After release, wrinkles were generated on the surface of the PDMS foam exposed to plasma. 
Characterization of PDMS foam with wrinkles. The surface morphologies and structural properties of wrinkles in the 3D PDMS foam were examined using field emission SEM (Hitachi, S-4300). The specific surface areas of the 3D PDMS foam were measured using X-ray micro-CT (SKYSCAN 1173, Bruker). To investigate the mechanical properties of the 3D PDMS foam, tensile tests were performed using a micro-fatigue tester (E3000LT, Instron).

FEA simulation. The strain distribution and deformation of the 3D PDMS foam were calculated using the structural mechanics module in COMSOL Multiphysics 5.4. The 3D geometry model was constructed based on the X-ray micro-CT (SKYSCAN 1173, Bruker) image obtained by scanning the 3D PDMS foam, but the geometry was miniaturized to oneeighth because of the computational resources. To mimic the application of uniaxial tensile strain, we prescribed displacement of one side of the 3D PDMS foam with a fixed opposite side using the linear elastic material model ${ }^{1}$. The displacement and stress distribution during the 3D motion was calculated by solving the following balance equations $\nabla \cdot\left(S+\varepsilon_{e l}\right)+F v=0, \varepsilon_{e l}$ $=\varepsilon-\varepsilon_{\text {inel }}, \varepsilon=\frac{1}{2}\left[(\nabla u)^{T}+\nabla u\right]$ where $S$ is von Mises stress, $\varepsilon_{e l}$ is elastic strain tensor, $F$ is body force per unit volume, $v$ is Poisson's ratio of material, $\varepsilon$ is strain tensor, $\varepsilon_{\text {inel }}$ is inelastic strain tensor, $u$ is displacement field, and $T$ is transpose. The equations are solved using stationary boundary conditions with a dense sampling of the prescribed displacement of 3D PDMS foam. To avoid numerical error in the scanning 3D image modeling, we re-meshed the 3D PDMS foam using the Autodesk Meshmixer with tetrahedron elements.

Analysis on a wrinkling model in 3D foam. To explain how the structural properties, such as wavelength and amplitude, of wrinkle architectures can be tailored for 3D foam, a wrinkling model for the 3D foam was applied by introducing the modified strain localized and concentrated on struts. In the wrinkling process, the elastic inner PDMS layer of struts in the 3D PDMS foam is likely to be compressed in order to return to the original state when the external tensile strain is removed. On the other hand, the stiff oxidized layer formed on the surface of the tensile-deformed struts tends to be bended not compressed. The total energy $\left(\boldsymbol{U}_{\boldsymbol{t o t}}\right.$ ) in the struts of the 3D foam for wrinkling can be presented as the sum of the bending energy of the oxidized layer and the strain energy of the inner PDMS layer..$^{2-4}$ As a result, the wrinkling model could be derived from minimization of the total energy $\left(\frac{\partial \boldsymbol{U}_{t o t}}{\partial \mathbf{A}}=\frac{\partial \boldsymbol{U}_{t o t}}{\partial \lambda}=\mathbf{0}\right)$ as follows ${ }^{2-4}$ : 


$$
\begin{gathered}
\lambda=\frac{2 \pi h}{\left(1+\varepsilon_{m}\right)\left(1+\frac{5}{32} \varepsilon_{m}\left(1+\varepsilon_{m}\right)\right)^{1 / 3}}\left(\frac{\overline{E_{s}}}{3 \overline{E_{i}}}\right)^{1 / 3} \\
A=\frac{h}{\sqrt{\left(1+\varepsilon_{m}\right)}\left(1+\frac{5}{32} \varepsilon_{m}\left(1+\varepsilon_{m}\right)\right)^{1 / 3}} \sqrt{\frac{\varepsilon_{m}}{\varepsilon_{c}}-1} \\
\varepsilon_{C}=\frac{1}{4}\left(\frac{3 \overline{E_{i}}}{\overline{E_{s}}}\right)^{\frac{2}{3}}
\end{gathered}
$$

where $\overline{\boldsymbol{E}}$ is the plane-strain modulus, calculated as $\overline{\boldsymbol{E}}=\boldsymbol{E} /\left(\mathbf{1}-\boldsymbol{v}^{\mathbf{2}}\right) ; \boldsymbol{E}$ is the Young's modulus; $\boldsymbol{v}$ is the Poisson ratio; $h$ is the thickness of the glassy surface layer; $\boldsymbol{\varepsilon}_{\boldsymbol{c}}$ is the strain when wrinkling structures start to be generated on the struts and $\boldsymbol{\varepsilon}_{\boldsymbol{m}}$ is the modified strain localized on struts. The subscripts $s$ and $i$ refer to the glassy surface layer and pristine inner layer of the struts, respectively. The oxygen plasma treatment equipment (PDC-32G Cleaner, Harrick Plasma, NY, USA) was applied to the PDMS foam, set up with a power of $18 \mathrm{~W}$, ultimate total pressure of $0.27 \mathrm{mbar}$, and RF frequency of $8-12 \mathrm{MHz}$. Therefore, the elastic modulus of the hard oxidized PDMS layer of the wrinkled 3D foam was determined as $40 G \boldsymbol{P a}$. For the analysis on the wrinkling model for 3D foam, we assumed $\overline{\boldsymbol{E}_{\boldsymbol{i}}}=\mathbf{2 . 1} \boldsymbol{M P a}\left(\boldsymbol{E}_{\boldsymbol{i}}=\mathbf{1 . 6}\right.$ $\left.M P a, v_{i}=0.5\right), \quad \overline{E_{s}}=43 G P a\left(E_{s}=40 G P a, v_{s}=0.27\right)$ and $\varepsilon_{c}=0.0007(0.07 \%) .^{5}$

In addition, the thickness of the oxidized layer $(h)$, a direct parameter to control the wavelength and amplitude of wrinkles according to the Eq. (1) and Eq. (2), can be controlled by adjusting plasma exposure time. Its thickness can be derived from the oxidation reaction kinetics. 5,6 During oxidation of the strut surface by plasma, methyl groups of the PDMS strut are substituted with hydroxyl groups. Some of the hydroxyl groups are converted to oxide links according to temporal and spatial variation. Specifically, the plasma acts as an initiator in this chemical process, which can react with PDMS that is not converted to the oxide glass yet. The rate of PDMS conversion into oxide glass can be described by multiplying a plasma intensity and a proportion of remained PDMS that can participate in the reaction. Finally, the frontal glassy layer-formation model can be presented by applying boundary conditions to the rate of PDMS conversion proportion (1. Before plasma treatment, the conversion proportion is zero, 2. If the plasma-treating time and space is infinite, the conversion proportion is one) as following 5,6 : 


$$
h=\frac{\ln (p \times t)}{\mu}-\frac{1}{\mu} \ln \left(\frac{1}{K I_{0}} \ln \left(\frac{1}{1-\Phi_{c}}\right)\right)
$$

where $t$ is the plasma exposure time, $\mu$ is the material attenuation coefficient by hindering and depleting ionic and radical oxygen, $p$ is the power applied in the plasma chamber, $K$ is the overall reaction rate constant, $I_{0}$ is the plasma intensity at the top surface of the PDMS strut, and $\Phi_{c}$ is the threshold of a PDMS conversion proportion $\Phi$ required to form a sufficiently hard oxidized layer for wrinkling. For the wrinkling process of the 3D foam, the oxygen plasma treatment equipment was applied to the PDMS foam, set up with a power of $18 \mathrm{~W}$, ultimate total pressure of $0.27 \mathrm{mbar}$, and RF frequency of $8-12 \mathrm{MHz}$. For the analysis on the wrinkling model for 3D foam, we assumed $K=5.5 \times 10^{-3}, I_{0}=1, \mu=0.075$ and $\Phi_{c}=0.06$. $^{5,6}$

Performance analysis of electrodes with wrinkled 3D foam for supercapacitors. We fabricated electrodes for stretchable supercapacitors by applying wrinkled foam coated with conductive Pt nanoparticles. The Pt nanoparticles with a thickness of $30 \mathrm{~nm}$ were deposited on the foam using a sputter equipment (Dual Target Sequential Sputtering System, Q300T D, Quorum Technologies). The electromechanical properties of the foam electrodes were measured at a constant current of $0.1 \mathrm{~mA}$ using a source meter (PXIE-1073). Using a threeelectrode CHI $627 \mathrm{~b}$ system (CH Instruments, Austin, TX), ${ }^{7}$ the electrochemical properties confirmed the impact of the wrinkled foam as the electrode. The three-electrode system comprised a reference electrode $(\mathrm{Ag} / \mathrm{AgCl}), \mathrm{Pt}$ mesh as a counter electrode, and an electrolyte solution of $0.1 \mathrm{M} \mathrm{Na}_{2} \mathrm{SO}_{4}$ (Sigma Aldrich), applying a voltage window of $0-0.6 \mathrm{~V}$. $\mathrm{MnO}_{2}$ deposition on the wrinkled foam was applied to the $\mathrm{MnSO}_{4} \cdot 5 \mathrm{H}_{2} \mathrm{O}$-mixed electrolyte solution $\left(0.02 \mathrm{M} \mathrm{MnSO}_{4} \cdot 5 \mathrm{H}_{2} \mathrm{O}\right.$ (Sigma Aldrich) and $\left.0.2 \mathrm{M} \mathrm{Na}_{2} \mathrm{SO}_{4}\right)$ used in the three-electrode system by a potentiostatic technique, in which the applied voltage was $1.3 \mathrm{~V}$. We calculated the capacitances of the foam electrodes using the CV curves. From $C=I /(d V / d t)$, where $I$ is the average current, the single-electrode specific volumetric capacitance $\left(C_{s p}\right)$ was examined using the following equation: $C_{s p}\left[F \cdot \mathrm{cm}^{-2}\right]=2 C / L_{\text {electrode }}$, where $L_{\text {electrode }}$ is the volume of the used foam electrode. 
(a) Ni foam

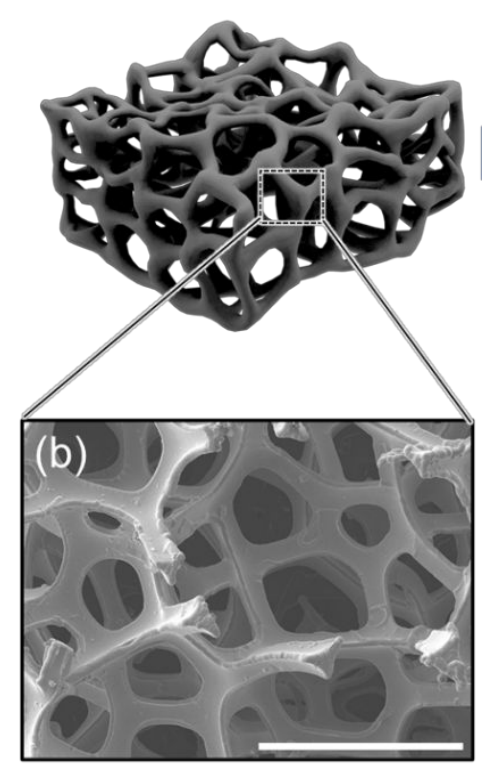

PDMS-coated Ni foam

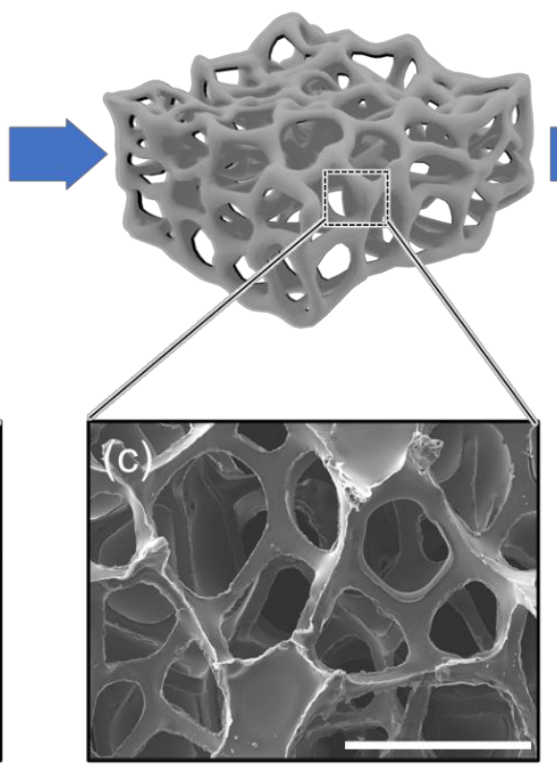

PDMS foam

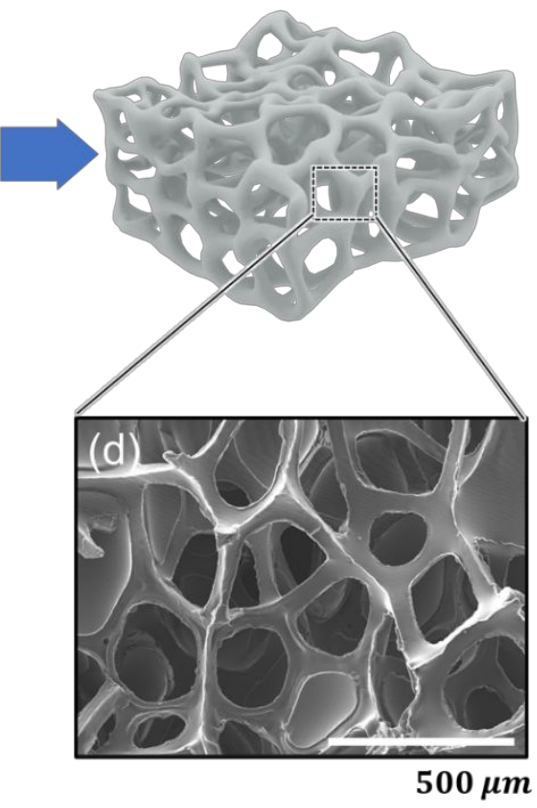

Figure S1. Fabrication of 3D PDMS foam (a) Schematic of the fabrication process. Elastic PDMS was coated onto rigid Ni foam, which serves as an interconnected 3D template, and then 3D PDMS foam was obtained after etching the inner Ni structure. SEM images of (b) Ni foam, (c) PDMS-coated Ni foam, and (d) 3D PDMS foam. 
(a)

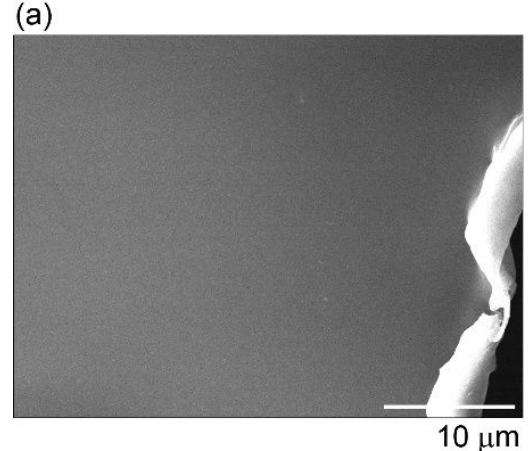

(b)

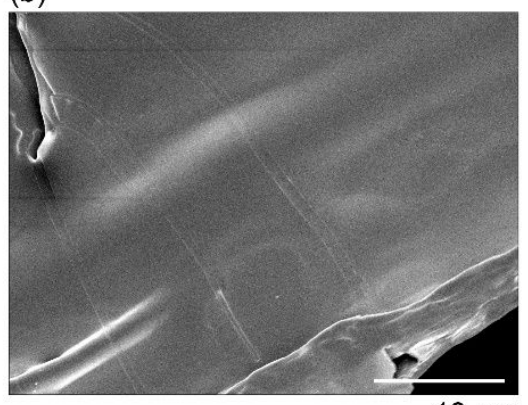

$10 \mu \mathrm{m}$ (c)

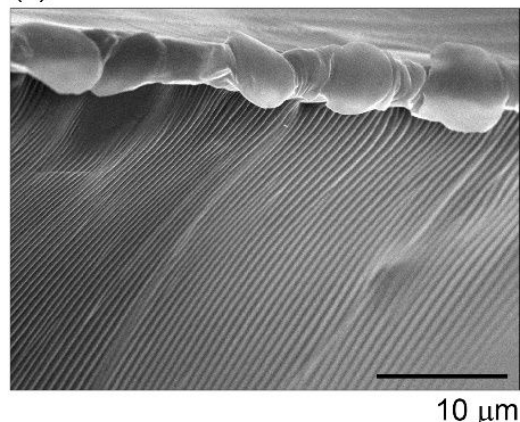

Figure S2. SEM images showing the surface of the struts in the PDMS foam treated with (a) only oxygen plasma, (b) only mechanical stretching-releasing, and (c) both plasma and mechanical strain. 
(a)

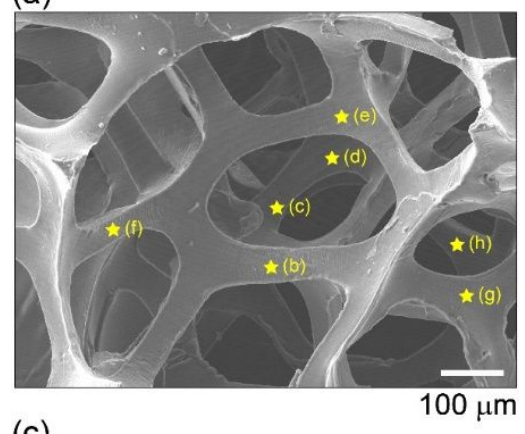

(c)

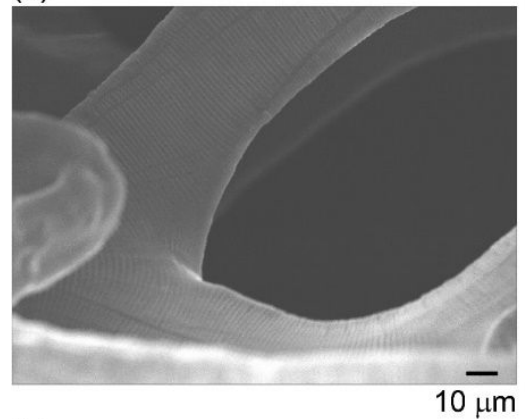

(e)

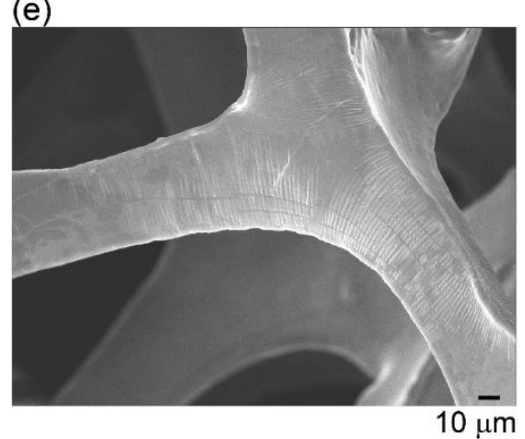

(g)

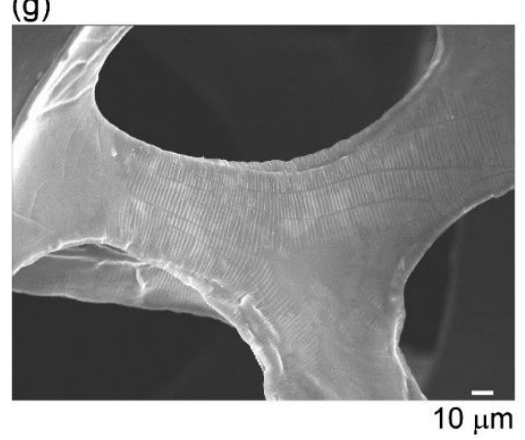

(b)

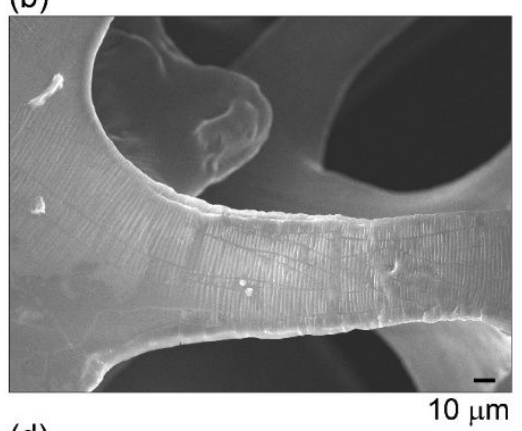

(d)
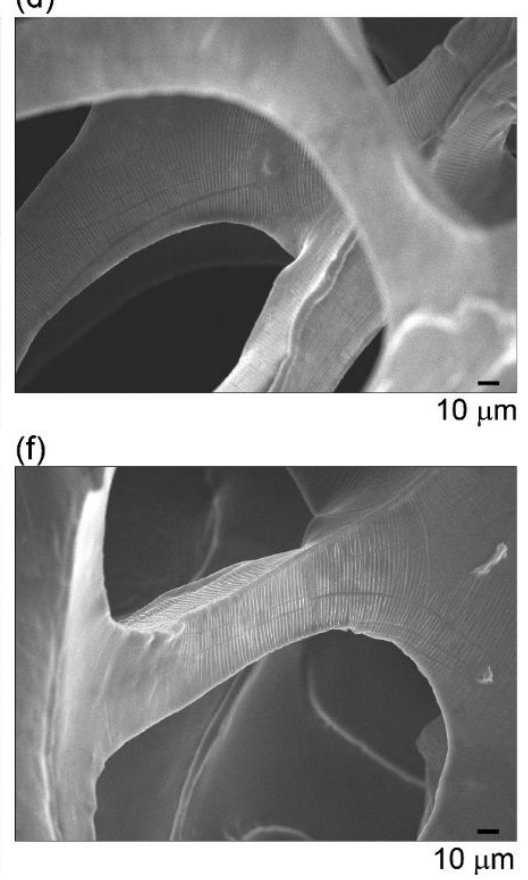

(h)

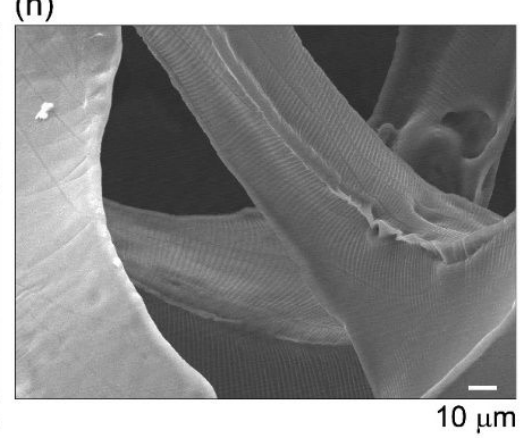

Figure S3. Wrinkle structures formed on the surfaces of the 3D PDMS foam after being exposed to plasma for an hour and stretching to $40 \%$ simultaneously. (a) SEM image of wrinkled 3D foam. (b-h) Enlarged SEM images at marked sites in (a). 


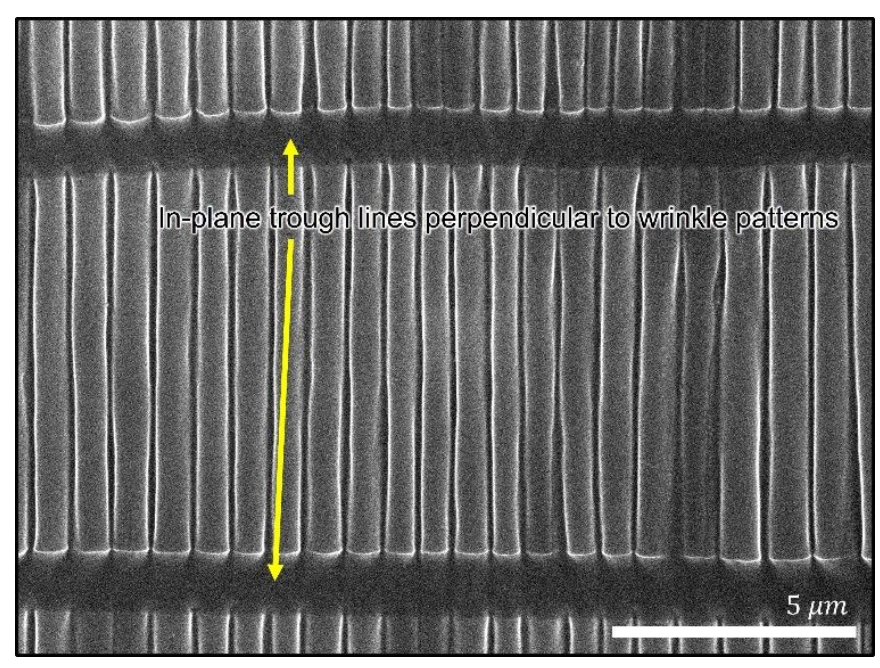

Figure S4. SEM image for in-plane trough lines perpendicular to wrinkle patterns on the strut surface of the 3D PDMS foam due to the Poisson effect of elastic PDMS struts under tensile strain. 
(a)

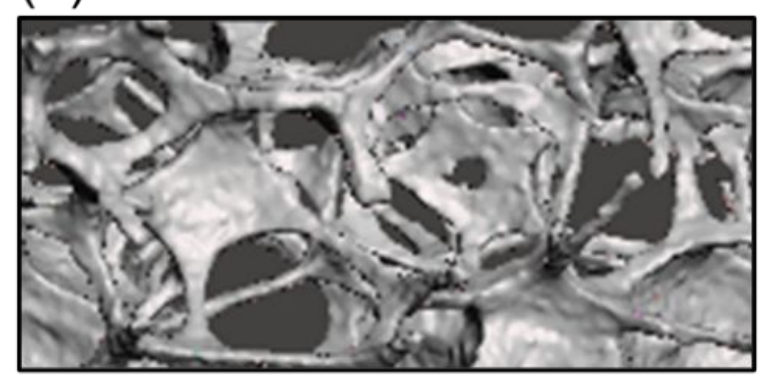

(b)

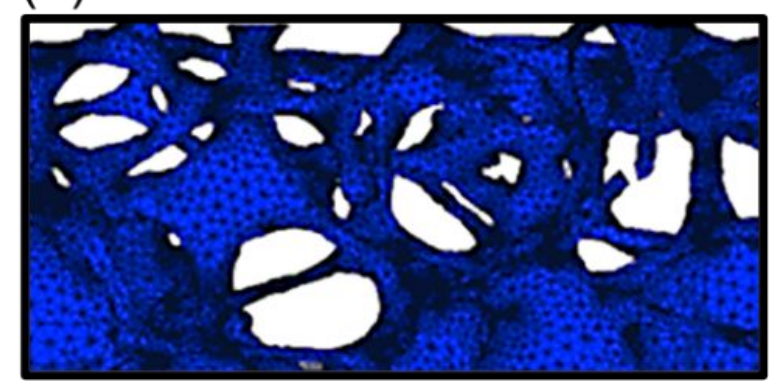

Figure S5. X-ray micro-computed tomography image of the 3D PDMS foam. 
(a)

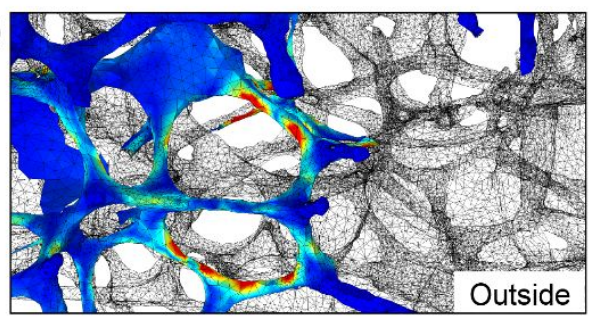

(b)

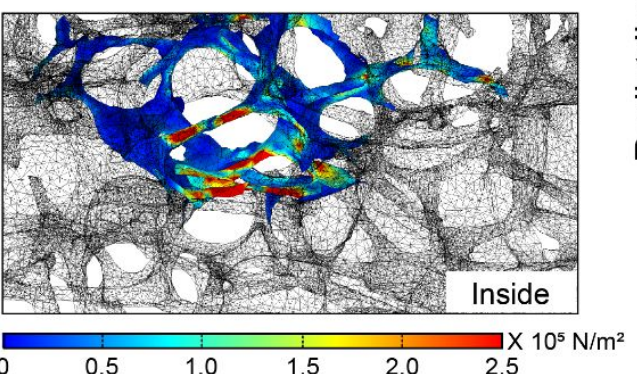

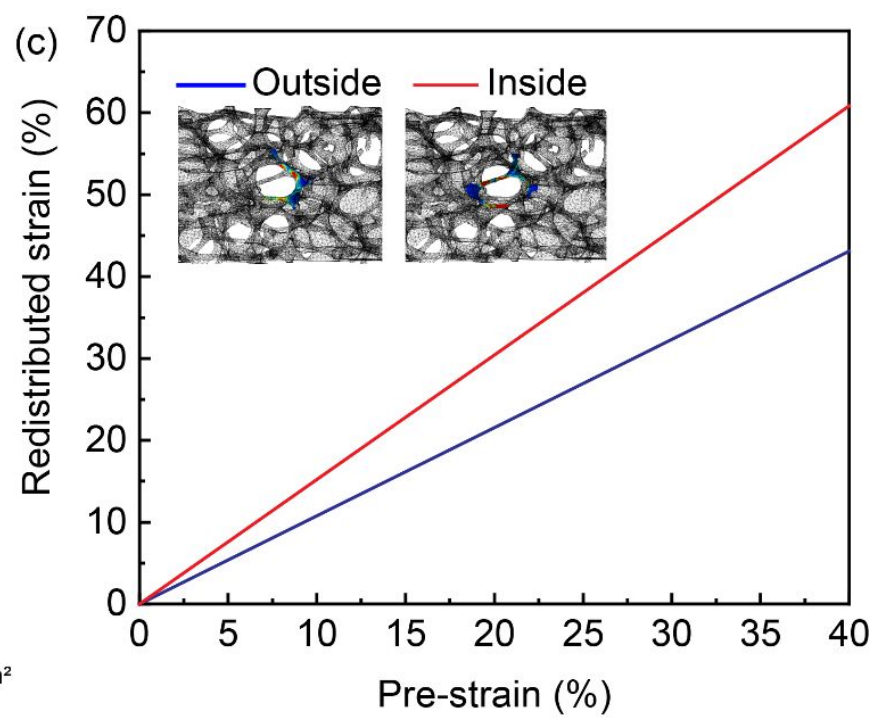

Figure S6. Tensile stress distribution on (a) outer and (b) inner struts of the 3D PDMS foam based on FEA simulation when the strain of $40 \%$ was applied. (c) Redistribution strain on the inner and outer strut surfaces of the 3D PDMS foam versus applied pre-strain. 


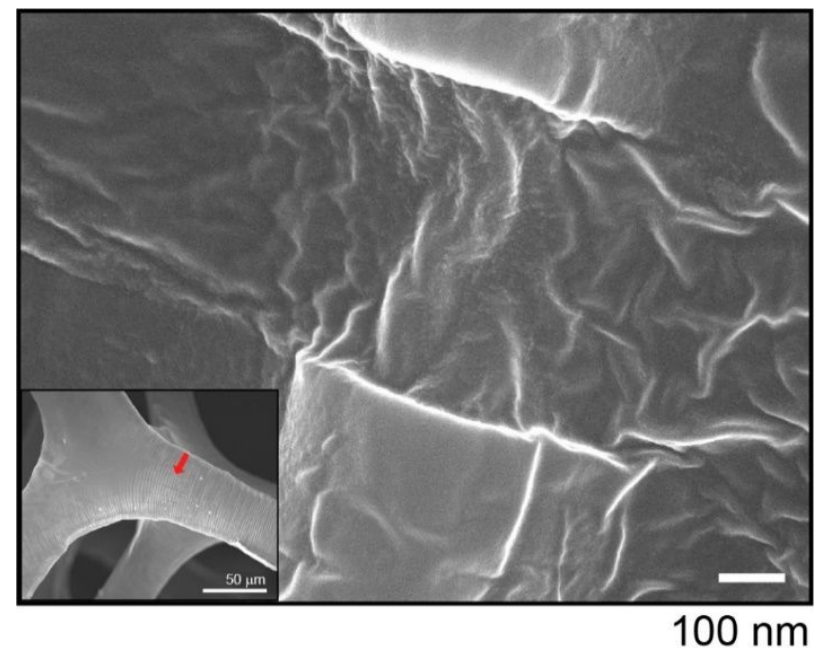

Figure S7. Surface wrinkle structure formed on PDMS surface. 

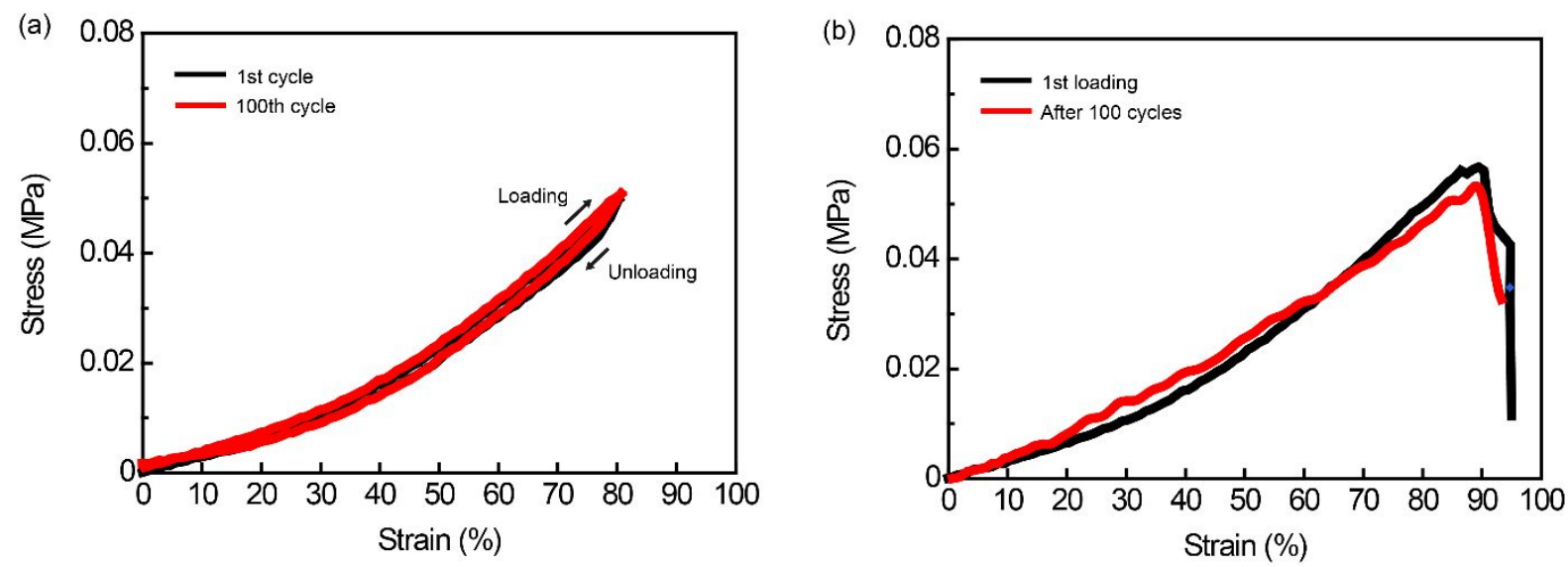

Figure S8. Stress-strain curves of the wrinkled 3D PDMS foam (pre-strain 40\%, plasma 1h) after stretching and releasing repeatedly. (a) A loading/unloading test when $80 \%$ strain was applied repeatedly. (b) Comparison between the initial stress-strain curve and a stress-strain curve after 100 stretching/releasing cycles. 


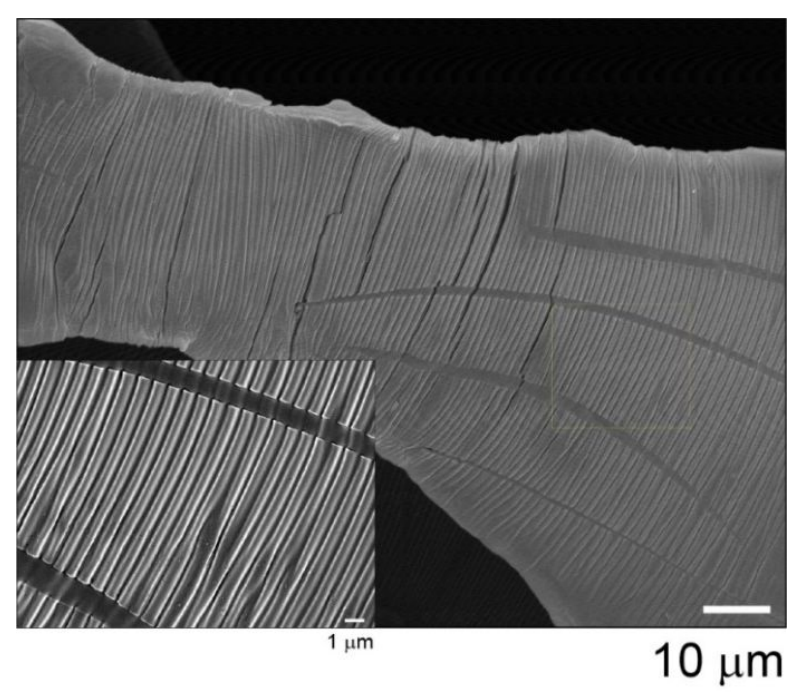

Figure S9. SEM image showing the conductively coated wrinkled 3D foam (pre-strain 40\%, plasma 1h) electrode. 


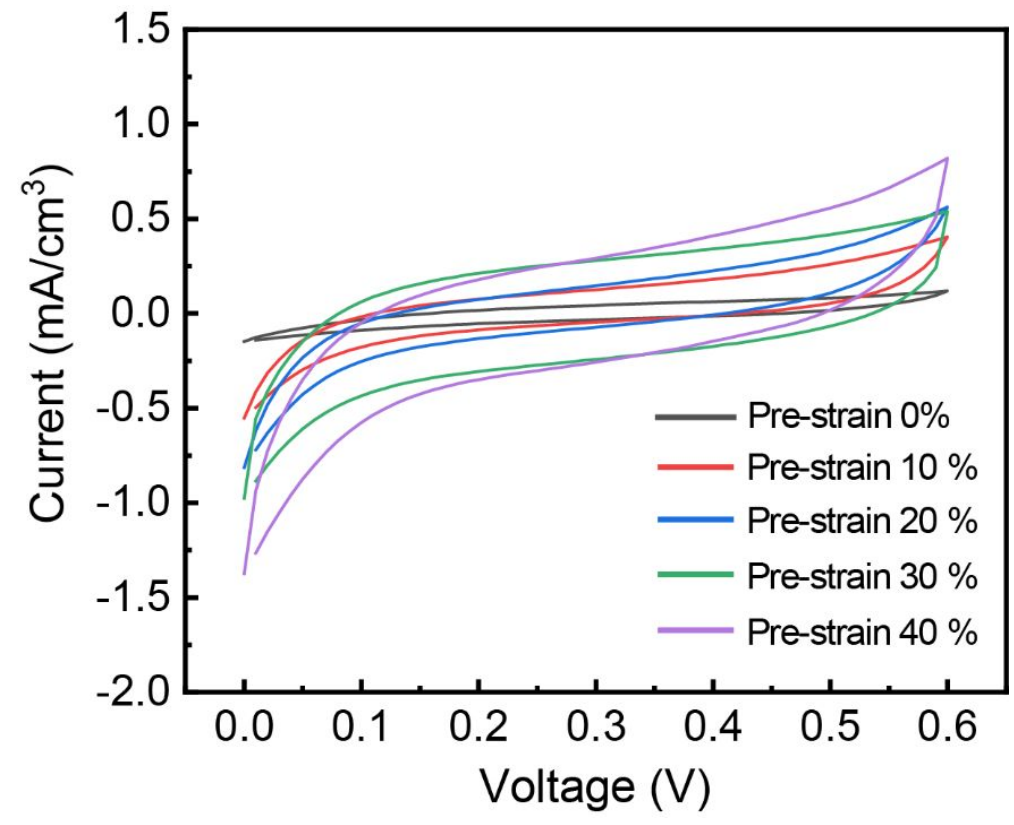

Figure S10. CV curves measured with the scan rate of $10 \mathrm{mV} / \mathrm{s}$ for the wrinkled 3D foam electrodes that were fabricated by exerting different pre-strains. The wrinkled 3D foam electrodes are made by same plasma treatment time of 1 hour. 


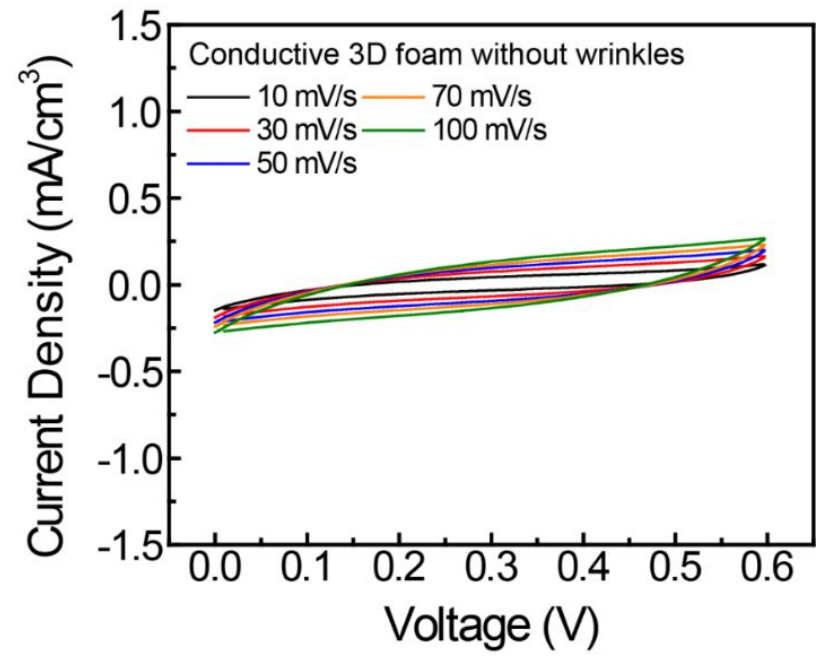

Figure S11. CV curves measured with scan rates of $10-100 \mathrm{mV} / \mathrm{s}$ for the conductive 3D foam without wrinkles. 
(a)

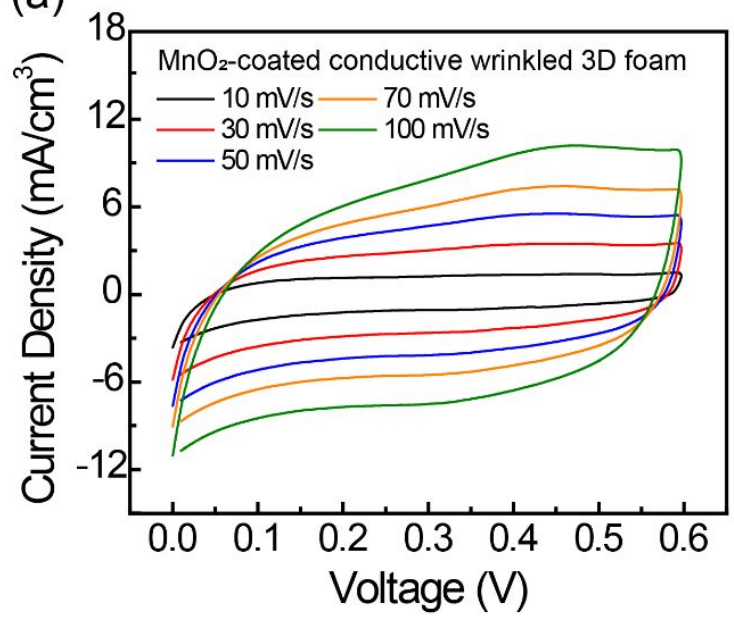

(b)

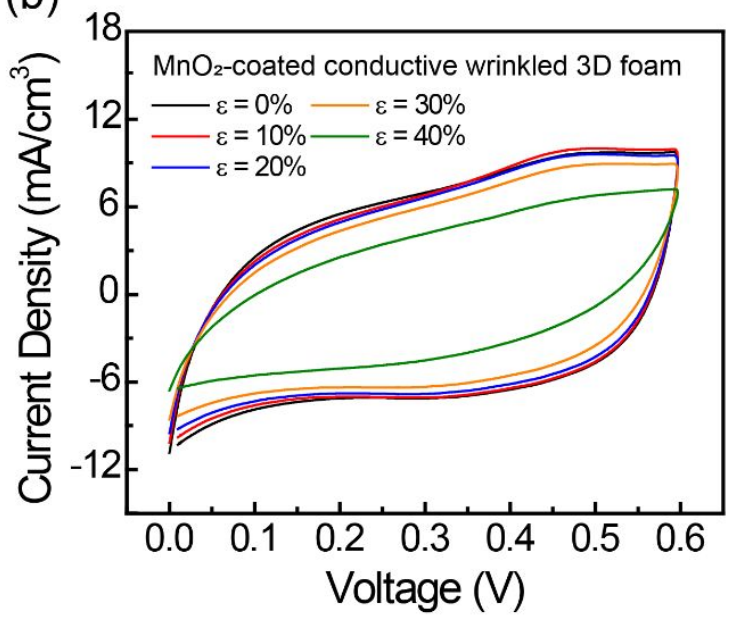

Figure S12. CV curves of $\mathrm{MnO}_{2}$-coated conductive wrinkled 3D foam supercapacitor. (a) CV curves measured with scan rates of $10-100 \mathrm{mV} / \mathrm{s}$. (b) $\mathrm{CV}$ curves measured with increasing strain $(0 \%-40 \%)$ in the tensile direction. 


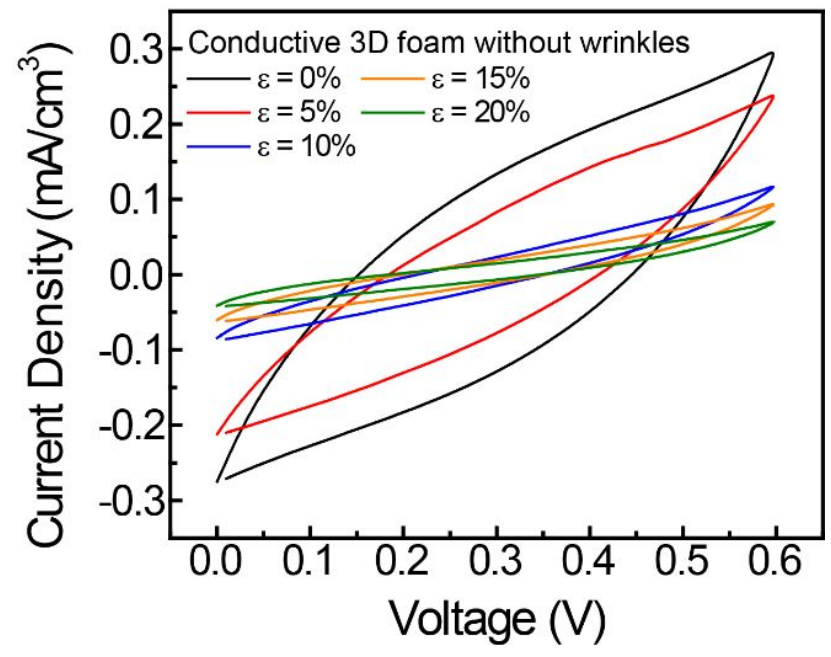

Figure S13. CV curves measured with increasing strain in the tensile direction, with tensile strains ranging from $0 \%$ to $20 \%$ for the conductive 3D foam without wrinkles. The scan rate remained the same at $100 \mathrm{mV} / \mathrm{s}$. 


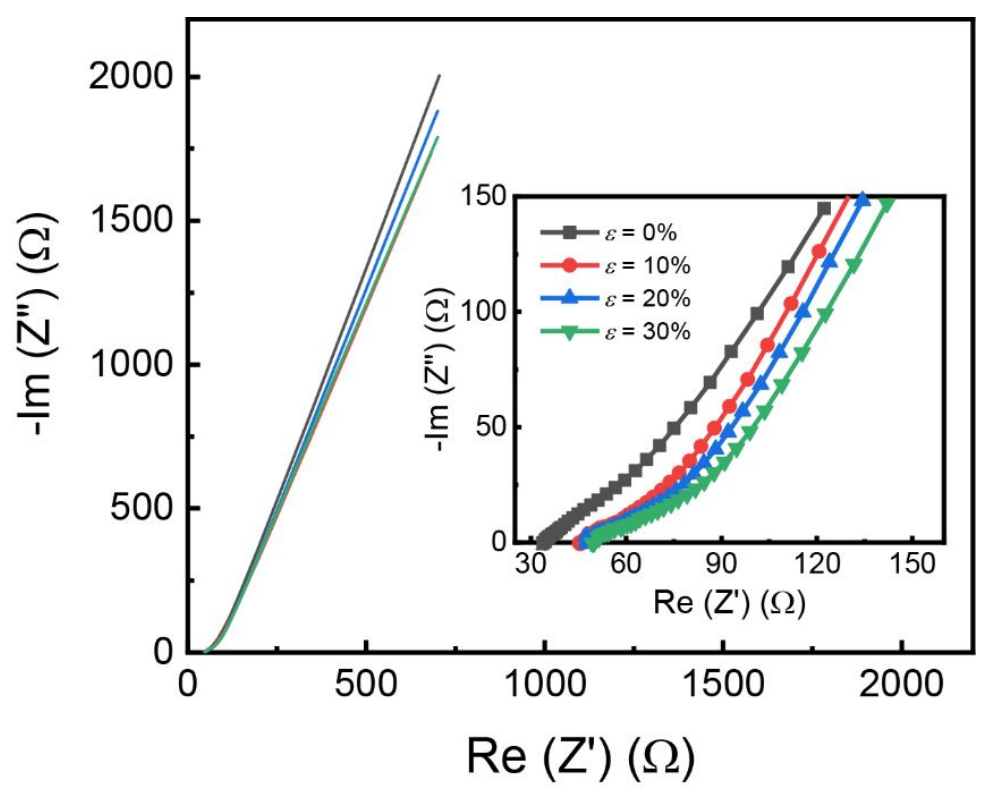

Figure S14. Nyquist curves of the wrinkled 3D foam electrode. The curves were measured with increasing strain $(0 \%-30 \%)$ in the tensile direction for the frequency range from 0.05 to 1000 $\mathrm{kHz}$ (the inset shows the high-frequency region). 


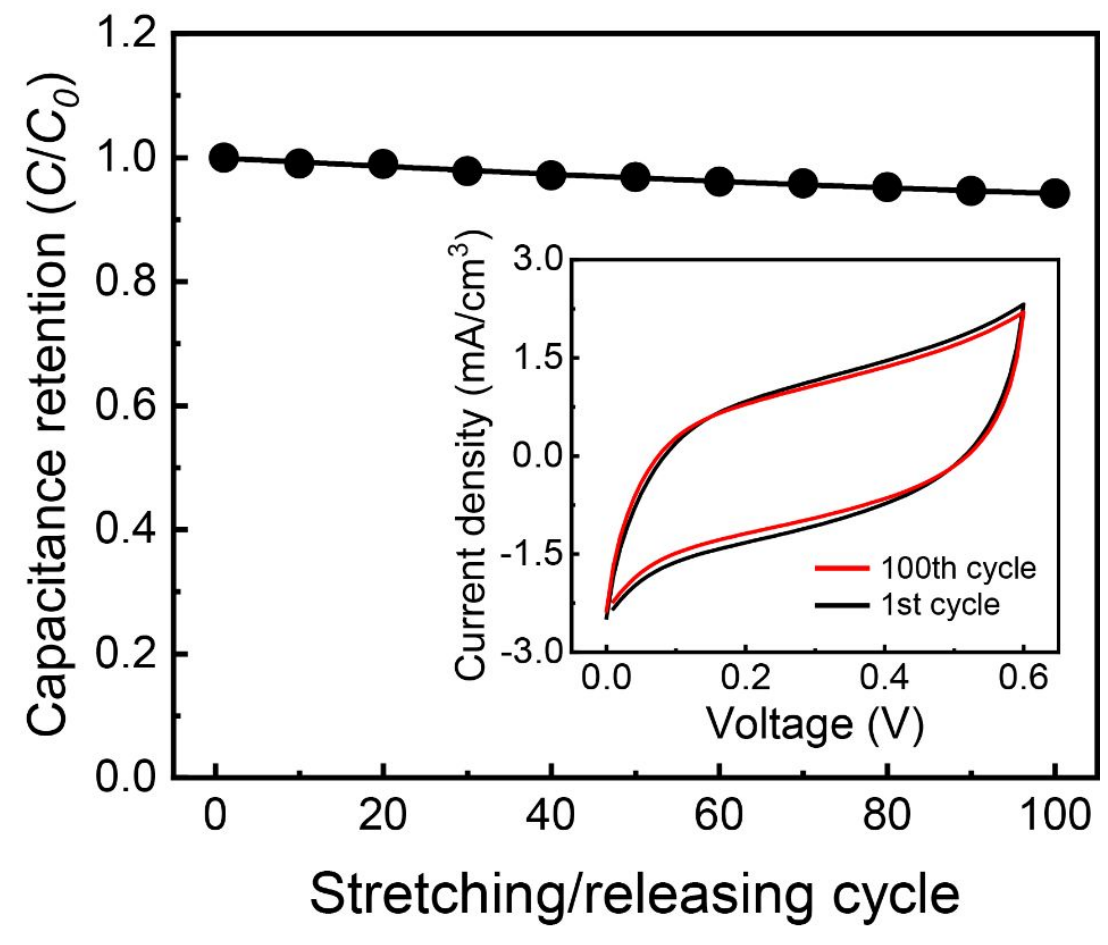

Figure S15. Capacitance retention versus mechanical stretching/releasing cycles up to $50 \%$ strain for the wrinkled 3D foam (pre-strain 40\%, plasma 1h) electrode. The inset shows CV curves at $100 \mathrm{mV} / \mathrm{s}$ scan rate before and after 100 times stretching/releasing cycles. 


\section{References}

(1) Tang, Z.; Gao, Z.; Jia, S.; Wang, F.; Wang, Y. Graphene-Based Polymer Bilayers With Superior Light-Driven Properties For Remote Construction Of 3D Structures. Adv. Sci. 2017, 4, (5), 1600437.

(2) Jiang, H.; Khang, D.-Y.; Song, J.; Sun, Y.; Huang, Y.; Rogers, J. A. Finite Deformation Mechanics In Buckled Thin Films On Compliant Supports. Proc. Natl. Acad. Sci. 2007, 104, (40), 15607-15612.

(3) Huang, R. Kinetic Wrinkling Of An Elastic Film On A Viscoelastic Substrate. J. Mech. Phys. Solids 2005, 53, (1), 63-89.

(4) Huang, Z.; Hong, W.; Suo, Z. Nonlinear Analyses Of Wrinkles In A Film Bonded To A Compliant Substrate. J. Mech. Phys. Solids 2005, 53, (9), 2101-2118.

(5) Bayley, F. A.; Liao, J. L.; Stavrinou, P. N.; Chiche, A.; Cabral, J. T. Wavefront Kinetics Of Plasma Oxidation Of Polydimethylsiloxane: Limits For Sub- $\mu \mathrm{m}$ Wrinkling. Soft Matter 2014, $10,(8), 1155-1166$.

(6) Nania, M.; Matar, O. K.; Cabral, J. T. Frontal Vitrification Of PDMS Using Air Plasma And Consequences For Surface Wrinkling. Soft Matter 2015, 11, (15), 3067-3075.

(7) Xie, Y.; Ju, Y.; Toku, Y.; Morita, Y. Fabrication Of $\mathrm{Fe}_{2} \mathrm{O}_{3}$ Nanowire Arrays Based On Oxidation-Assisted Stress-Induced Atomic-Diffusion And Their Photovoltaic Properties For Solar Water Splitting. RSC Adv. 2017, 7, (49), 30548-30553. 